\title{
Mitigation of traffic disaster by analyzing motorist's movement behavior based on an image captured by a drone
}

\author{
Yosritzal $^{1^{*}}$, Purnawan $^{1}$, Merry Lovita ${ }^{2}$, and Dilum Dissanayake ${ }^{3}$ \\ ${ }^{1}$ Center for Transportation, Civil Engineering Department, Andalas University, Indonesia \\ ${ }^{2}$ Civil Engineering Department, Andalas University, Indonesia \\ ${ }^{3}$ School of Engineering, Newcastle University, UK
}

\begin{abstract}
Uncontrolled motorists' movement behavior could trigger a disaster for road users. A deep understanding of the motorist's movement behavior might improve our mitigation planning. This paper presents an observation of the movement of motorists from a minor road crossing a major road at an unsignalised intersection. The study's objectives are to estimate the gap and lag of motorcyclists when crossing the intersection, observe the forced gap, and predict the effect of traffic volume on the variation of the gap and lag. The case study was located at the intersection between Ahmad Yani Road as a major road and Bandar Purus Road as a minor road in Padang, Indonesia. A drone was used and placed vertically above the center of the intersection to record the movement. The study found that the average lag is 2.4 seconds at off-peak, and the average gap is 4.3 seconds; at the peak hour are 2.0 seconds and 3.1 seconds, respectively. At peak hours, more than $80 \%$ of motorcyclists are committed to the force gap/lag.
\end{abstract}

\section{Introduction}

Motorist movements at an intersection play an important role when it comes to traffic safety and related problems. Especially when the intersection is unsignalised, this requires meticulous attention. At unsignalised intersections, the priority sign or give way sign controls vehicle maneuver and movement. However, motorists tend to disobey the sign in some cases due to knowledge limits and personal [1]. As a result, it is expected that at the unsignalised intersection, traffic problems such as congestion and traffic accidents. The disobeying traffic rule will be a disaster for road users and must be mitigated before it grows uncontrollably.

Motorcyclists are the most vulnerable road users, as shown by traffic data from Indonesia [2]. In most traffic accidents, motorcyclists are usually involved, either as a victim or a perpetrator. Fig 1 shows traffic accidents in the first half-year of 2017, where the number of motorcyclists involved in the accident is more than 31,000. In his report [3] found that from $100 \%$ of accidents, about $89 \%$ of them involved a motorcyclist.

Compared to any city globally, Padang is among the cities with the highest percentage of motorcyclists. The 2016 traffic data shows that more than $75 \%$ of the traffic in Padang are motorcyclists [4]. Each month, the number of new motorcycles registered in Padang reaches 600 units [5]. The high number of motorcyclists is also the case at the national level, where $81.3 \%$ of the traffic in
2013 was motorcycles. The annual growth rate of motorcycles is about $7.5 \%$ [6].

Researchers should have a deep understanding of motorists to be able to mitigate traffic accidents. One of the critical situations is at an unsignalised intersection.

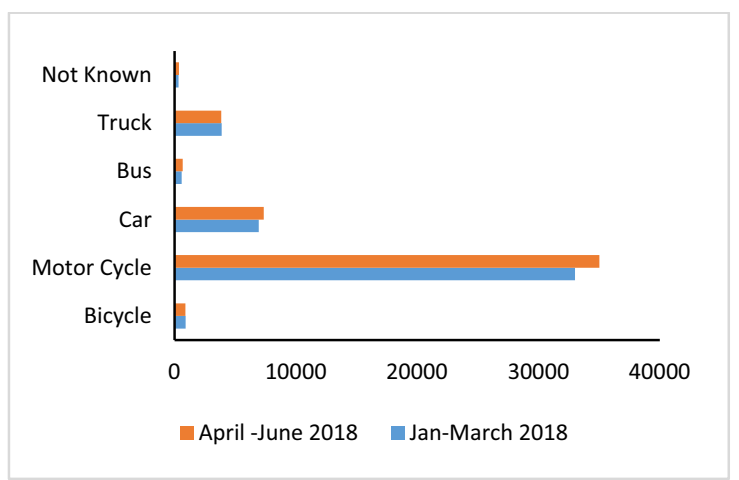

Fig. 1. The occurrence of accidents [2]

The research found that at unsignalised intersections, usually, five seconds is a critical gap for a vehicle to cross [7]. However, a study in Padang shows that the critical gap was pushed to a minimum as motorcyclists from a minor road tend to take a risk to interrupt the straight direction traffic at the major road to allow them to cross [8]. The 2016 study reported that as the gap is forced to a minimum, the straight direction vehicles must push the brake lever to avoid an accident [8].

* Corresponding author: yosritzal@eng.unand.ac.id 
As the forced gap became a phenomenon in Padang, a more intensive study was conducted and reported in this paper. The study captured the movement of a motorcyclist from a minor road crossing a major road at an unsignalised intersection.

The study aims to identify the forced gap occurrence at an unsignalised intersection where motorcycles from a minor road cross a major road in Padang. The gap and lag between the passing vehicles and the incoming vehicles were observed to identify the forced gap. The terminology of a gap is defined as the interval of time when a vehicle passes a pointer to the following vehicle passes the same point [9]. Another terminology for the gap is a gap acceptance, defined as the safe gap perceived by a motorist to cross the road. Perhaps, the most relevant terminology for the interval of time for a motorist to cross a low-volume traffic road is lag. Lag is the time difference needed for an approaching vehicle from a major road to reach the crossing point at the intersection and a vehicle from a minor road to the same point. Fig 2 shows the illustration of the gap, and Fig 3 shows the illustration of the lag.

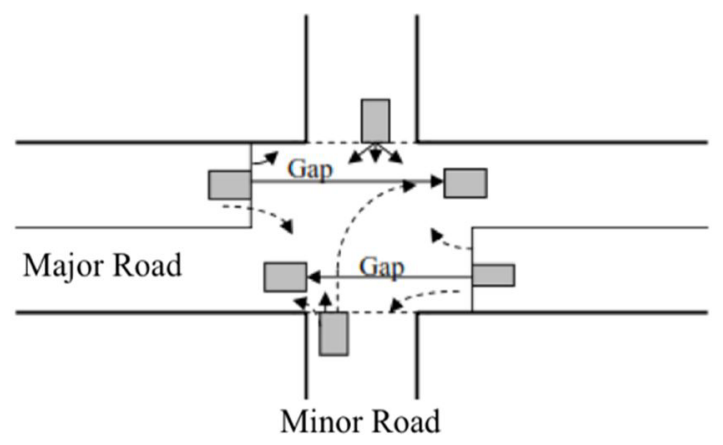

Fig. 2. Illustration of the gap [10]

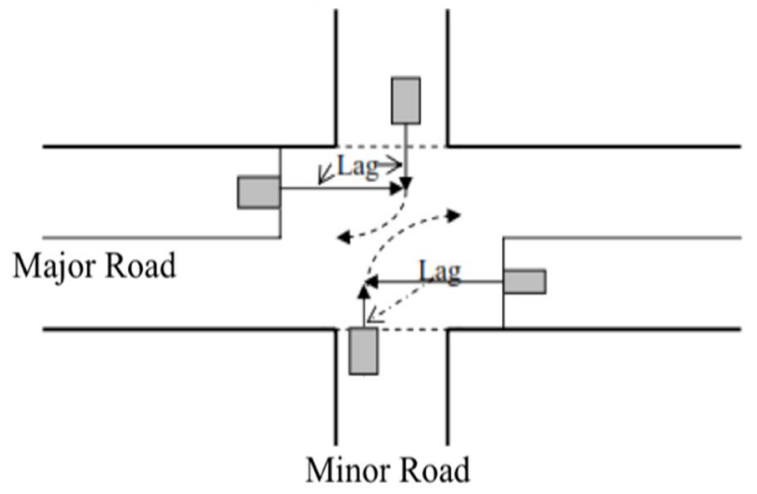

Fig. 3. Illustration of lag [10]

\section{Review of Relevant Literature}

\subsection{Application of drone technology in engineering application and practice}

A drone is a small unmanned aerial vehicle (UAV) guided by remote control or computer. Drones have cameras to take photos from different angles. The use of a drone in engineering project has some key benefits such as safer access to sites which is difficult to access by human such as a high place, working over water or steep slopes; faster and cost-effective for collecting data from a large site; and easy to integrate with GIS and CAD software [11]. The Klohn Crippen Berger has used a drone for a slope stabilization project along a river in British Columbia. The drone has also been used to inspect a landslide next to a highway.

Tkac and Mesaros [12] also highlight the use of a drone in various civil engineering fields, especially in the construction industry such as building surveys, topography mapping and land surveys, construction site inspection, equipment tracking and automating, remote monitoring, and progress reports, integration of laser scanning and aerial photogrammetry and thermal imaging recording. The use of a drone in civil engineering has many benefits[12], such as: creating real-time aerial images, overview reveals assets and challenges and also it is allowed planners to meet virtually to discuss project timing, equipment needs and challenges presented by the terrain.

\subsection{The use of drone technology in transport planning}

The use of drones in transportation engineering has been found in some literature, such as the American Planning Association, which uses drones to support transportation planning by air support [13]. Similarly, [14] explores the possibilities to use a drone in transportation planning, designing, and monitoring. However, the most shared articles discuss drones as air transportation to distribute logistics such as [15] and [16].

\section{Methodology}

In this study, some data related to the geometric of the studied intersection and traffic were collected. Manual measurement was conducted to take the geometric data, including the lane width, number of lanes, the wide of pedestrian or road shoulder. The traffic-related data was collected from a pre-recorded video of the scene from a drone. Compared to other methods, a drone might be more reliable because it has many advantages, such as allowing the camera to record the scene vertically from above at the desired height, and the recorded video can be played as many times as needed.

The drone used in this study was a quadcopter type, namely DJI Phantom 4. The DJI Phantom 4 has a 180degree rotatable and high-resolution camera to capture live video or still images. The high capacity battery and GPS make the drone event better as it could record the live video for approximately 20 minutes each time at the exact point. The detail about the DJI Phantom 4 can be found on the DJI website [17], whilst the effective use of a drone in traffic observation was highlighted in [8].

This study was conducted in Padang, West Sumatra, Indonesia. An unsignalised intersection in a commercial area was the object of the study. The major road is A Yani road, and the minor one is Bandar Damar Road with a Bandar Purus Road at the opposite, as shown in Fig 5. 


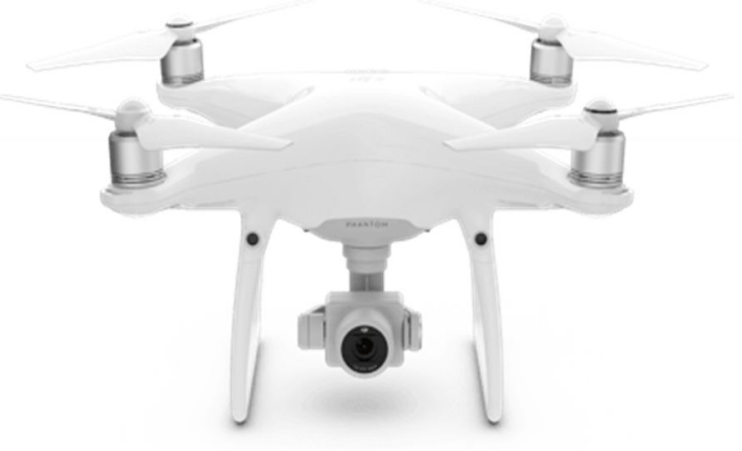

Fig. 4. Typical of DJI Phantom 4 [17]

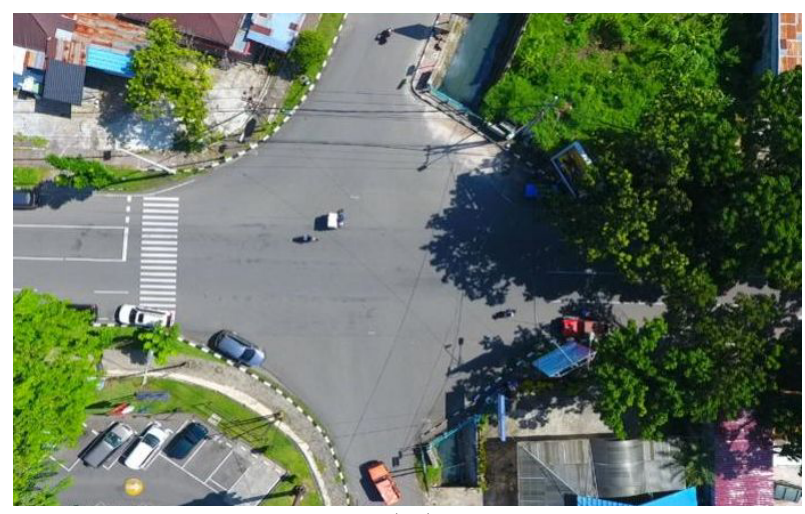

( a )

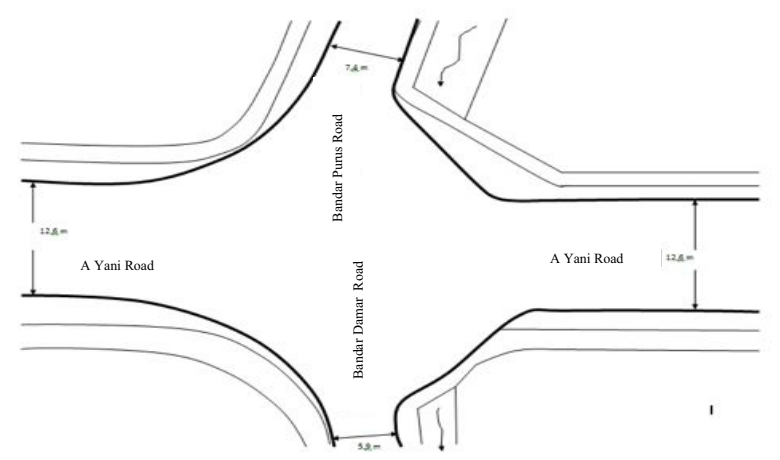

(b)

Fig. 5. ( a ) Image of the location from a drone and ( b ) twodimensional model of the intersection.

The intersection is formed by a $12.6 \mathrm{~m} \mathrm{~A}$ Yani Road and a $5.9 \mathrm{~m}$ Bandar Damar Road with a $7.4 \mathrm{~m}$ Bandar Purus Road at the opposite side of the Bandar Damar Road. At the time of the observation, there was no traffic sign on both sides of the minor road. Usually, at the unsignalised intersection, there is a "Stop" sign or a "Yield" sign. Fig 6 shows the intersection from the Bandar Purus Road heads to Bandar Damar Road.

As the drone only capture the video for approximately 20 minutes, the observation will be conducted three times, the first one was at the peak hours, the second one at the medium traffic volume (medium peak hours), and the third one at the low traffic volume (off-peak hours). To know the interval time of the peak, medium, and low traffic volume, a traffic count survey was conducted to record the traffic volume at the major road from 06.00 to 18.00. Based on the recorded video, a traffic counting was

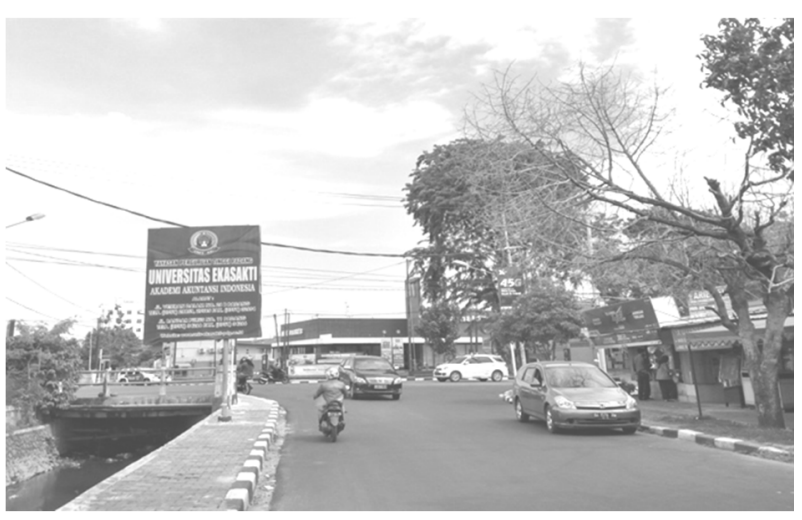

Fig. 6. Ground image of the intersection

conducted to obtain the hourly traffic volume, as shown in Fig 7. The peak hour was observed between $14.30-$ 16.00 with a traffic volume was at least $2500 \mathrm{pcu} / \mathrm{h}$. The medium traffic volume was observed between 09.0014.00 , with traffic volume was between $2000-2500 \mathrm{pcu} / \mathrm{h}$. Traffic volume less than $2000 \mathrm{pcu} / \mathrm{h}$ was observed between 06.00-07.30. Based on the traffic variation, the drone observations were conducted two times in the offpeak time interval, two times in the medium peak interval, and two times in the maximum peak time interval.

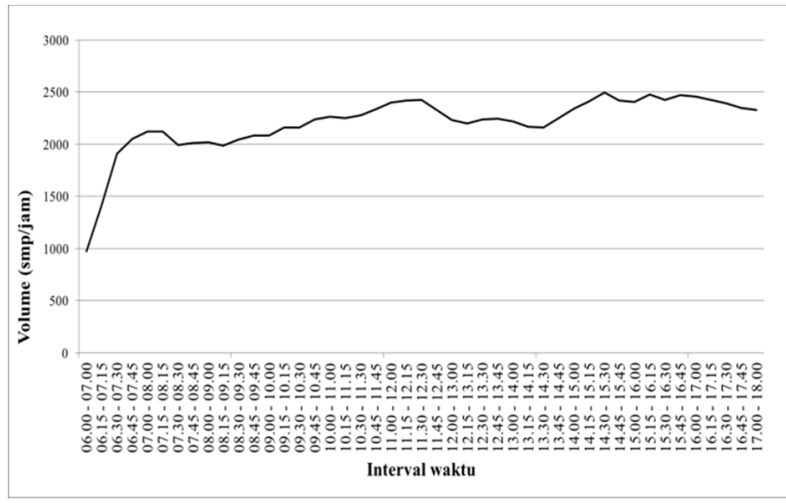

Fig. 7. Observed Traffic Volume

\section{Results}

\subsection{Off-Peak Period Case}

Low traffic volume was observed during the off-peak period, where the volume was less than $2000 \mathrm{pcu} / \mathrm{h}$. As the traffic volume was low, and the density was low as reasonably. Considering that the speed and the density are inversely proportional, it is expected that the speed increases with decreasing the density. However, in this study, the vehicle speed was not estimated.

Fig 8(a) shows the lag in seconds at the off-peak period. On average, the lag is about $2.4 \mathrm{~s}$, and the standard deviation is $0.8 \mathrm{~s}$. Fig $8(\mathrm{~b})$ shows the gap in second at the off-peak period. The mean gap is $4.3 \mathrm{~s}$, and the standard deviation is $1.1 \mathrm{~s}$. Comparing the lag and the gap, we found that the lag is lower than the gap allegedly caused by the rare vehicle observed. From Fig 8, the forced gap is expected to happen when the lag is minimal. Fig 9 
shows the moment when the forced gap occurs at the offpeak.

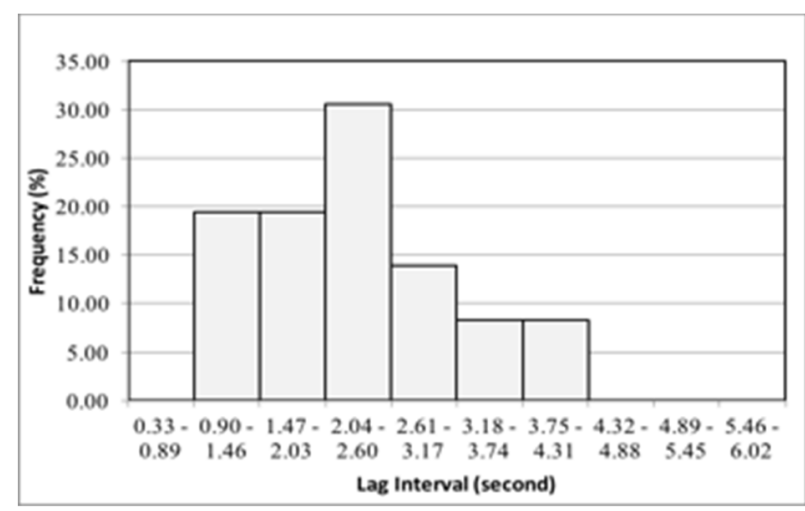

( a )

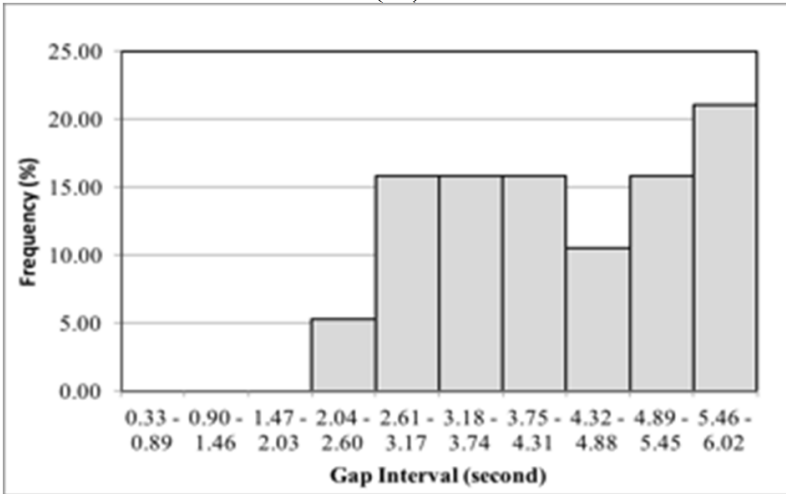

(b)

Fig. 8. The lag and gap at off-peak ( a ) Lag histogram and (b) Gap histogram

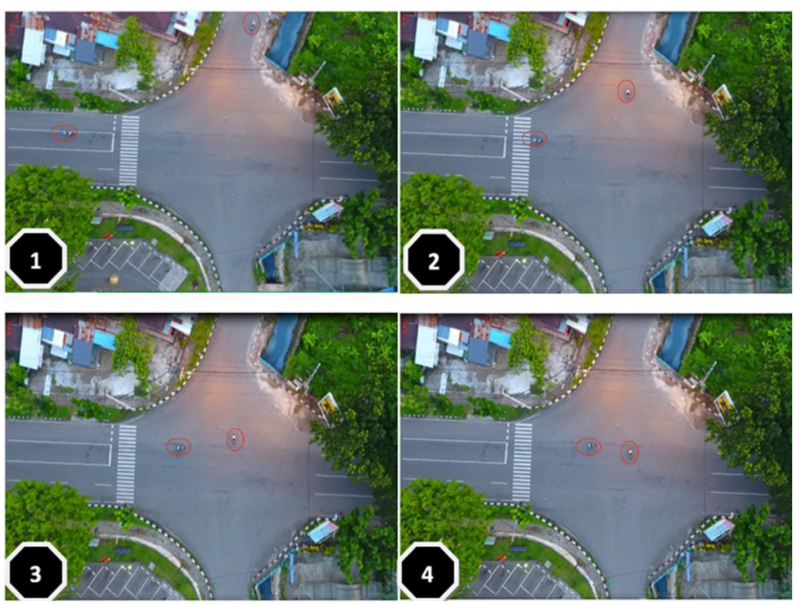

Fig. 9. Occurrence of Forced Gap during Off-Peak Period

\subsection{Medium Peak Period Case}

Compared to the off-peak period, the traffic volume in the medium peak period is a beat higher in the medium peak, so the density is also higher. With about $2000 \mathrm{pcu} / \mathrm{h}$ to $2500 \mathrm{pcu} / \mathrm{h}$ traffic volume, the mean of lag was recorded at $2.1 \mathrm{~s}$, and the standard deviation was $0.8 \mathrm{~s}$. The mean gap was $3.3 \mathrm{~s}$, and the standard deviation was $3.3 \mathrm{~s}$. Fig 10(a) shows the histogram of lag, and Fig 10(b) shows the histogram of the gap. Fig 11 shows the occurrence of the forced gap at the medium peak period where the motorist from a minor road pushed the gap, which might lead to an accident if the vehicle on the major road did not push the brake or reduce their speed.

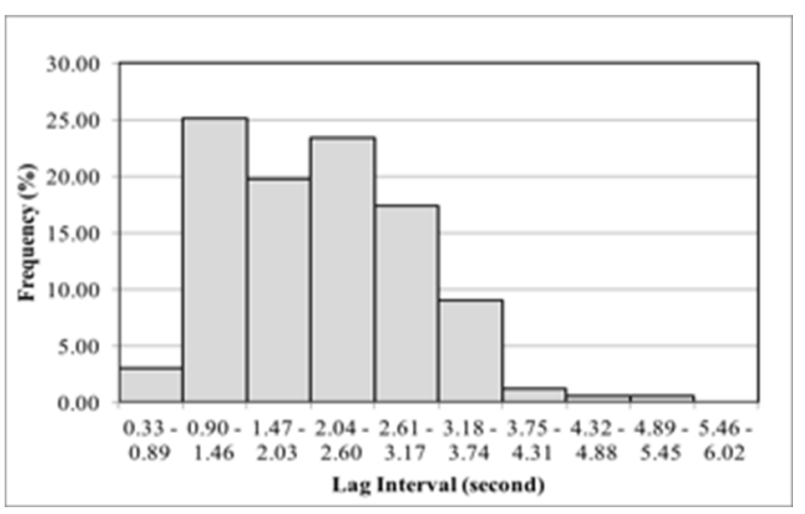

( a )

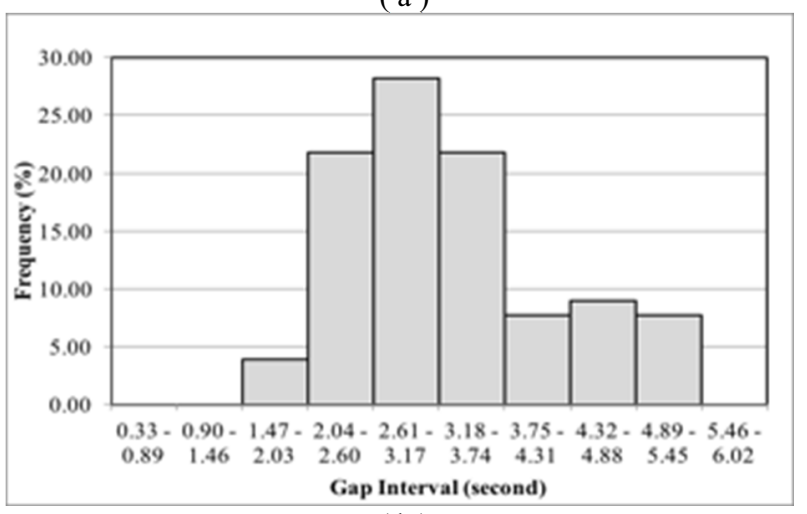

( b )

Fig. 10. The lag and gap at off-peak ( a ) Lag histogram and (b) Gap histogram

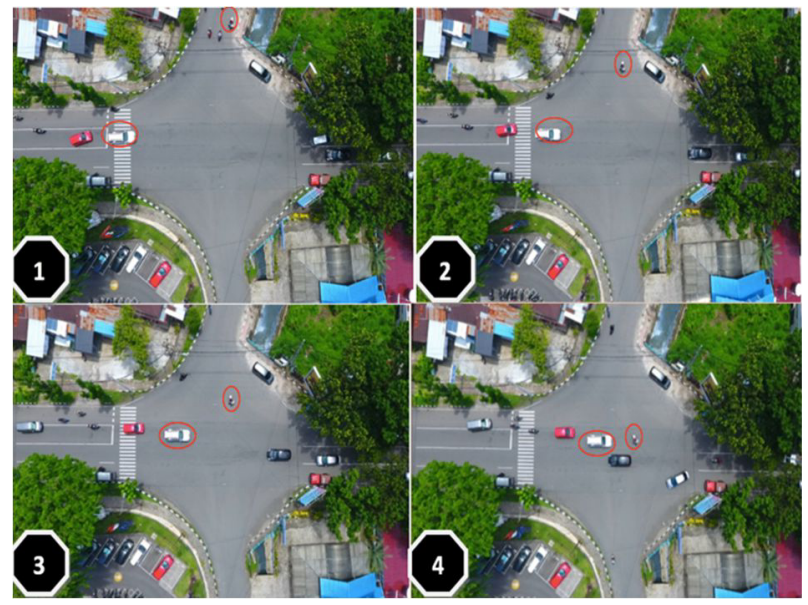

Fig. 11. A brave motorist forces the gap at Medium-peak Period

\subsection{Maximum Peak Period Case}

During the maximum peak period, with traffic volume increasing, the density also increases, but the speed reduces. The lag and gap also reduce as the speed reduces. The mean of lag was $2 \mathrm{~s}$, and the standard deviation was $0.6 \mathrm{~s}$, whilst the mean lag was $3.1 \mathrm{~s}$, and the standard deviation was $0.7 \mathrm{~s}$, as shown in Fig. 11(a) and (b). It was observed that in the maximum peak period, the lag and gap were shorter, and the motorists risky their life by 
crossing the road without waiting for a safe lag or gap, as shown in Fig 12.

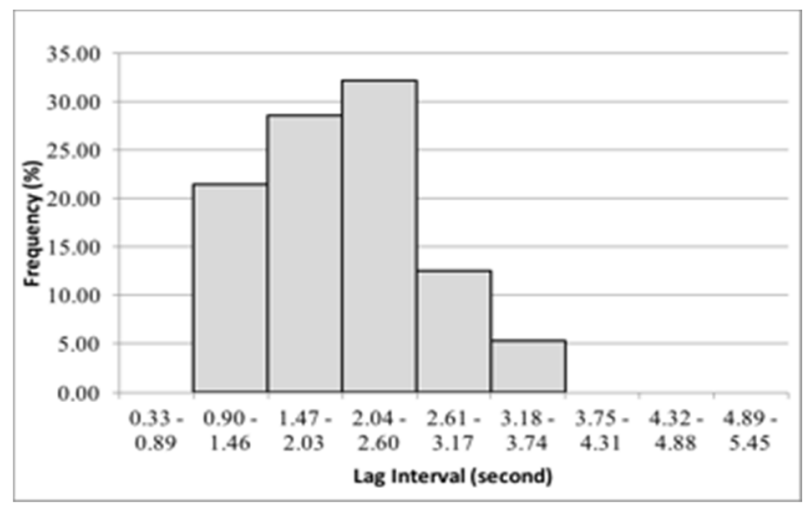

( a )

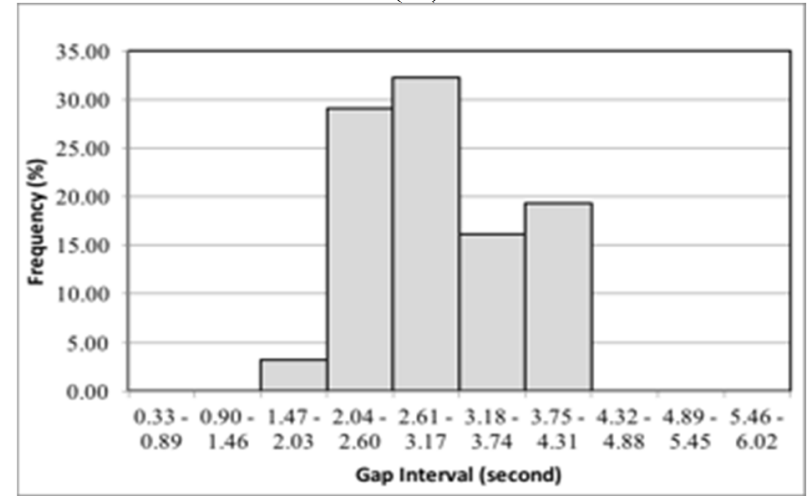

(b)

Fig. 11. Lag and Gap observed during Maximum-Peak Period

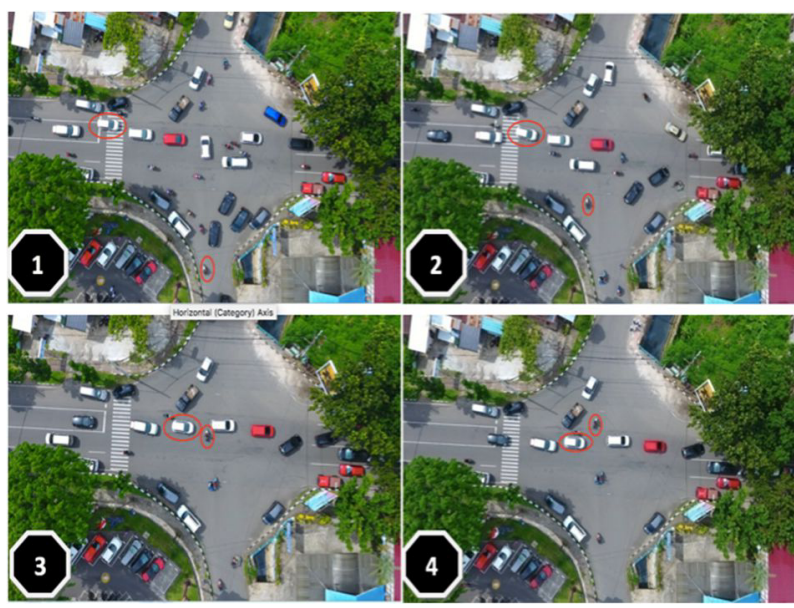

Fig. 12. Risky movement of a motorcyclist at Maximum-Peak Period

\subsection{Discussion}

First of all, we would like to highlight that the use of drones in data collection in this study has helped us obtain more accurate data than using a camera recorder at the roadside. The accurate data is very promising as the technology of drones proliferates. When this study was conducted, the drone's battery only lasted for about 20 minutes to keep the drone in the air. It is expected that in the future, the operating time of drone might be improved as the battery last longer.
Secondly, we will discuss the results of the study. Data in sections 4.1 to 4.3 indicated that the lag and the gap were shorter with increased traffic volume. The gap and lag found in this study are shorter than found in other studies on an international basis, such as [18] in Ahmedabad. Furthermore, as the mean of gap acceptance in Padang is $3.4 \mathrm{~s}$ [19], we can assume that about $69.44 \%$ of motorists were forced the lag and about $5.26 \%$ tend to force the gap because the gap and lag are shorter than average. The occurrence of the forced gap by motorists increases with the increasing traffic volume, increasing traffic density, and decreasing the average speed of traffic. Minimum gap and lag occur when the maximum traffic volume indicates that the motorists tend to push the gap and lag to gain access crossing the major road. It is rarely the case for the traffic at the major road to voluntarily access traffic from a minor road to cross at the peak hour. Concern should be raised for traffic safety in this situation. When the forced gap becomes a trend among motorists, it will be a disaster for all traffic users. The life of the motorists relies on the awareness and reaction of other drivers. An accident could be avoided if the other drivers were aware and immediately pushed the brake when a motorist forced the gap. Unfortunately, it is not safe at all as the driver behind might not have a chance to push their brake to hit the vehicle in the front. Another possibility is when the motorist forced the gap, and the other driver has no chance to push the brake, they might swerve to the left or the right with the risk of hitting or being hit by other vehicles.

With safety as our priority, the authority needs to reduce the forced gap and lag at the unsignalised intersection. On the other hand, the interest in crossing vehicles from the minor road is also essential to accommodate. Public advertisements regarding traffic safety should be displayed continuously and improvement of traffic management and engineering at the intersection, and taking action of the law enforcement against those who disobey the safety rule.

\section{Conclusions}

The results of the motorist's movement observation at an unsignalised intersection in Padang have been presented. An essential result of the study was that motorcyclists tend to push the gap and lag when traffic at the major road is heavy. With an average lag of $2.1 \mathrm{~s}$ at the maximum peak period, the motorist's risk involved in an accident is high. A traffic accident is a disaster for the big family of the victim, especially when the victim is the backbone of the family. Therefore, we recommend the authority to reduce the possibility of a motorist pushing the gap by implementing education, engineering, and law enforcement policy.

Furthermore, to the best of the author's knowledge, the application of drone technology to investigate this issue is new and has never been addressed the points in detail before. This study has proven that the use of drones could be beneficial for more accurate data collection. Our future work would be analyzing the data using microsimulation software to increase the accuracy of our prediction. 
The authors would like to express their great appreciation to the Civil Engineering Department of Universitas Andalas, who provide funding for the publication of this research with contract number: 134/UN16.09.D/PL/2021.

\section{References}

1 W. Santosa, Sukarno, JRS, 3 (1), 64-76 (2013)

2 Korlantas POLRI, Statistik Laka (Statistic of Traffic Accident) (2018)

3 S.K.A. Singh, I.S. Nasution, L. Hayati, MKS, 47 (2), 105-109 (2015)

4 B.P.S, Number of Vehicle by Type in Padang, (2018)

5 Y. Arham, New Vehicle Growth in Padang Dominated by Motorcycle (2018)

6 B.P.S. Growth of Motor Vehicles by Type, 19492016 (2018)

7 A. Suraji. WT, 19 (1), 5-11 (2011)

8 Yosritzal, F. Auliani, Analysis of forced gap and gap acceptance of motorcylists, in the Proceeding of $3 \mathrm{rd}$ ACE National Conference, Jurusan Teknik Sipil, Universitas Andalas, 22-23 Oktober 2016. Universitas Andalas, Padang, Indonesia (2016)
9 J.L. Gattis, ST Low, JTE, 125(3) (1998)

10 J.E. Hummer. ITE. Prentice-Hall. inc (1994)

11 Klohn.com, Using Drones in Engineering Projects, (2018)

12 M. Tkac, P. Mesaros, JoCE, 14 (1), 27-37 (2019)

13 N. Veraart, D. Reel, A. McConnell, Air support for transportation planning (2020)

14 D. Cvitanić, "Drone applications in transportation," in Proceedings of the 5th International Conference on Smart and Sustainable Technologies (SpliTech), 1-4 (2020)

15 MARSH, Drones - a view into the future for the logistics sector? (2015)

16 ITF, Ready for Take-off? Integrating drones into the transportation system, International Transport Forum (2021)

17 https://www.dji.com/id/phantom-4-pro-v2/specs

18 AK Maurya, H.J. Amin, A. Kumar, TRP, 17, 203-212 (2016)

19 RL Vika, Undergraduate Thesis, Andalas University (2013) 